\title{
Correlation between Perceived Stress and Bruxism in Students
}

\section{Yamna Matin Afridi}

Department of Dentistry, Margalla Institute of Health Sciences, Pakistan

*Corresponding author: Yamna Matin Afridi, Department of Dentistry, Margalla Institute of Health Sciences, Dentistry, Quaid-e-Azam Avenue, Gulrez-III, Rawalpindi, Pakistan, Tel: +923365551721; E-mail: yamnamatinafridi@gmail.com

Received date: August 25, 2018; Accepted date: September 11, 2018; Published date: September 15, 2018

Copyright: $\odot 2018$ Afridi YM. This is an open-access article distributed under the terms of the Creative Commons Attribution License, which permits unrestricted use, distribution, and reproduction in any medium, provided the original author and source are credited.

\begin{abstract}
To find the correlation between stress and bruxism in students, a detailed analysis of stress and bruxism is done. Stress is one of the main triggering factors of bruxism and shows a strong correlation with bruxism and this is the main idea of this research conducted. This study shows gender-based co-relation between stress and bruxism by finding out the percentage of bruxism and stress in affected students. The study also points out the high ratio of bruxism with stress in the particular gender. The study is conducted on undergraduate students enrolled in a fouryear college program and the research concludes by comparing and relating the stress and bruxism of the students in these four years.
\end{abstract}

Keywords: Bruxism; Undergraduate; Stress

\section{Introduction}

Stress is a body's reaction to a challenge. There are four primary types of symptoms of stress; physical, emotional, cognitive and behavior [1]. Students experience more stress as compared to the general population [1]. Reference [1] studied the sources of stress in students and these sources were diagnosed to be change in sleeping habits, increase in workload, financial pressure and new responsibilities. Reference [2] concluded that females exhibit more stress as compare to males and the particular idea was also implemented in this paper. Emotional stress is the main triggering factor of bruxism $[3,4]$ and Stress has a great impact on the life of a student. This study shows the correlation between stress and bruxism in students as bruxism is characterized as one of the physical symptoms of stress [5]. Bruxism is Grinding and clenching of teeth, it's a parafunctional disorder with various etiologies $[6,7]$. The three main etiological factors of bruxism were the Occlusal, Pathophysiological and Psychological factor [8]. Occlusal factors include factors such as tooth interferences in dental occlusion, while Pathophysiological factors involve the release of brain neurotransmitter [8]. Certain medications like SSRIs (Serotonin Reuptake Inhibitor) and Amphetamine also lead to increase in bruxism [8]. Cigarette smoking leads to bruxism as well; smokers report bruxism two times more than non-smokers [8]. The Psychological factors such as anxiety and stress, which is common among students, leads to bruxism and this analysis on students, had been the focus of this paper. In another study [9], it was reported that Asian students have the highest incidence of stressrelated bruxism that is $24.6 \%$, as compared to students of EuroAmerican, African-American, and Hispanic background. This research had been conducted in Pakistan, a country in South Asia to find and relate to the percentage of bruxism and stress. In a gender-based analysis, women reported a higher level of stress as compared to males [10]. This research had also pointed out the gender-based effects of stress leading to bruxism in female students. Bruxism affects the quality of life through multiple dental problems such as attrition of teeth, cracks in dentition $[11,12]$, muscle and temporomandibular joint pain [13].
It displays two circadian manifestations; one is sleep bruxism and the other one is awake bruxism. The study we conducted showed the prevalence of bruxism, and its correlation with apparent stress in a group of Pakistan college students at Margalla Institute of Health Sciences (MIHS) was inspected.

\section{Methods}

A Cross-sectional study was conducted among 256 undergraduate students of Margalla Institute of Health Sciences with a group of 64 students each from the first year, the second year, third year and final year. Participants of the study were selected in an equal amount from different departments. Informed consent was taken, and the questionnaires were distributed among randomly chosen individuals on the campus (Table 1).

\begin{tabular}{|l|l|}
\hline Students & Numbers \\
\hline Male Students & 128 \\
\hline Female students & 128 \\
\hline Total no of students & 256 \\
\hline
\end{tabular}

Table 1: Number of participants.

The questionnaire elicited information on-

1) Demographic characteristics (age, gender)

2) Experience of grinding teeth during day and night time

3) Stress evaluation through the Perceived Stress Scale

Perceived Stress Scale (PSS), a classical stress assessment tool, was selected to measure stress in any individual as per standards [14]. Ten questions were given to the participant from PSS tool, and answers were given with 4 points on a scale on that.

- $\quad$ Never $(0)$

- Almost never (1)

- Sometimes (2) 
Page 2 of 3

- $\quad$ Fairly often (3)

- Very often (4)

The 10 questions asked targeted the stress in the last 4 weeks' time of participant. Scoring was given according to PSS scale from 0-40. A higher score indicated more perceived stress and lower score indicated less perceived stress. Whereas, Bruxism was investigated using the following questionnaire:

1) Do you grind or clench your teeth during sleep or has anyone heard you grinding your teeth when you sleep?

2) Do you grind or clench your teeth when you are awake or has anyone seen you grinding or clenching your teeth when you are awake?

3) Do you feel soreness or fatigue of jaw when you wake up?

4) Does the temple area of your head aches, when you wake up?

5) Have you noticed any worn-down dentition?

6) Do you have any difficulty opening the mouth?

The answers were scored on a Likert-type scale [13], ranging from "never" to "always", from 1 to 5. The demographic character questionnaire investigated the age and gender of the participant. The aim of these methods was to investigate the following:

- The occurrence of Bruxism in students at MIHS

- Prevalence of Stress in students at MIHS

- Correlation between perceived stress and bruxism in students at MIHS, Pakistan

- Gender-related association between stress and bruxism in students at MIHS, Pakistan

\section{Results}

The contributors of the study were divided into two groups of Males and Females. 128 males were selected, and 128 females were selected. The study further divided the group of males and females' students according to their year of study and selected 32 males and females each from 1st year, 2nd year, 3rd year and final year of MIHS.

Table 2 shows a correlation between stress and bruxism in male students in 4 years of the undergraduate program. In 1st year undergraduate students, $62.5 \%$ percent student exhibited stress with $30 \%$ of male students with stress showed the prevalence of bruxism and $25 \%$ male students showed the prevalence of bruxism without stress. In 2nd year undergraduate students, $56.25 \%$ of male students displayed stress, while $22.2 \%$ male students showed the prevalence of bruxism with stress and $14.28 \%$ showed bruxism without the stress factor.

\begin{tabular}{|l|l|l|l|l|l|}
\hline Year & Males & Stress & No Stress & Bruxism & No Bruxism \\
\hline 1st & 32 & 20 & 12 & 6 & 3 \\
\hline 2nd & 32 & 18 & 14 & 4 & 1 \\
\hline 3rd & 32 & 19 & 13 & 3 & 1 \\
\hline 4th & 32 & 26 & 6 & 7 & 1 \\
\hline
\end{tabular}

Table 2: Stress and bruxism in male student.
In 3rd year undergraduate male students, $59.3 \%$ of males exhibited stress with $15.7 \%$ males showing the prevalence of bruxism with stress and $15.38 \%$ showed bruxism without stress. In final year undergraduate students, the stress level in males was increased to $81.25 \%$ according to study. $26.9 \%$ of the students showed bruxism with stress, while $16.6 \%$ showed bruxism without stress. Altogether $64.8 \%$ male students showed stress and $24 \%$ showed bruxism along with stress and $15.5 \%$ showed bruxism without stress.

In Table 3, a correlation between bruxism and stress in female students is displayed. 128 female students were selected, and 32 female students were selected from each 1st year, 2 nd year, 3 rd year and final year of undergraduate studies at MIHS.

In the study conducted from 1st year undergraduate female students, $78.1 \%$ exhibited stress, while $48 \%$ of female students with the prevalence of stress-related bruxism and $28.5 \%$ with bruxism without stress.

\begin{tabular}{|l|l|l|l|l|l|}
\hline Year & Female & Stress & No Stress & Bruxism & No Bruxism \\
\hline 1st & 32 & 25 & 7 & 12 & 2 \\
\hline 2nd & 32 & 21 & 11 & 10 & 2 \\
\hline 3rd & 32 & 22 & 10 & 11 & 2 \\
\hline 4th & 32 & 28 & 4 & 12 & 1 \\
\hline
\end{tabular}

Table 3: Stress and bruxism in female student.

The study on 2nd year female student showed $65.6 \%$ female students in stress with $47.6 \%$ female students exhibiting stress-related bruxism and $18.1 \%$ showed bruxism without stress. In the 3rd year of study $68.7 \%$ female students showed stress, the percentage of female students with stress-related bruxism was $50 \%$, while female students who showed bruxism without stress was $20 \%$. In final year undergraduate female students, the stress level was comparatively high as compared to other year students. $87.5 \%$ of the female students exhibited stress. $50 \%$ of female students showed bruxism with stress and 25\% of female students showed bruxism without any stress factor. The altogether combined result of four years of female students showed $75 \%$ of female students exhibiting stress. $48.95 \%$ percent of female students had bruxism with stress and $21.8 \%$ showed bruxism without stress (Figures 1 and 2).

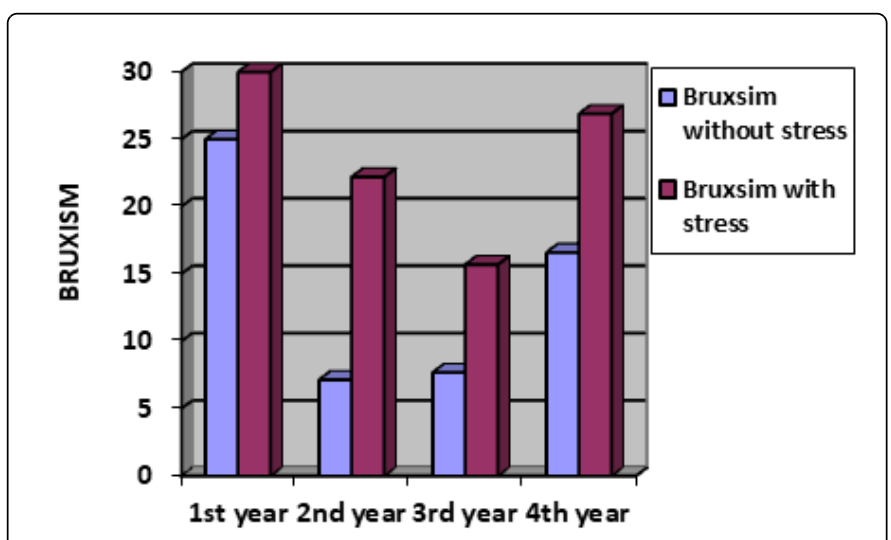

Figure 1: Correlation between bruxism and stress among males. 


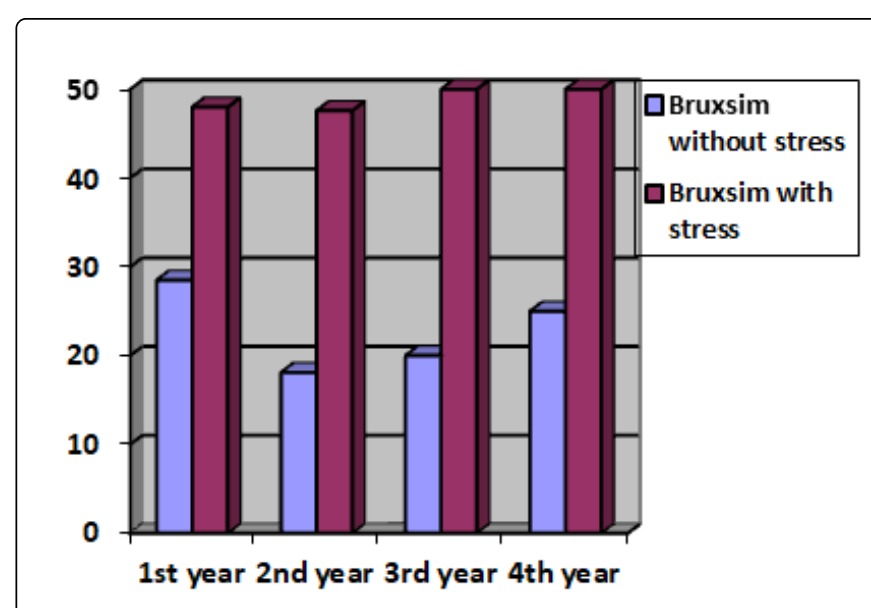

Figure 2: Correlation between bruxism and stress among females.

\section{Discussion}

College students are prone to stress. Some students face challenges being away from home as well, maintain the academic record, and economic crisis are one of the few factors that cause the stress aggravation in students.

The study conducted in this paper showed that bruxism was more prevalent in male and female students with perceived stress. The male students showed $24 \%$ prevalence of bruxism along with stress, while $15.5 \%$ showed bruxism without stress. In female's $48.95 \%$ of females had bruxism with stress, while $21.8 \%$ had bruxism without stress.

The finding proved the relationship of bruxism and stress as bruxism was more prevalent in students who exhibited stress.

The study also showed that female students showed more prevalence of bruxism as compare to the male students, $40.62 \%$ of female students complained of bruxism, whereas $20.31 \%$ of male students had bruxism.

The female students also showed a higher level of stress in the four years of undergraduate education as compare to the male students, $75 \%$ of female students exhibited stress in four years of study whereas 64.8\% male students exhibited stress. The result showed that female students had a high prevalence of stress as well.

In a survey in [14], it was demonstrated that stress reduction skills have an impact on reduction in bruxism, thus strengthening the argument that stress plays a vital role in bruxism.

\section{Limitations}

The study conducted has limitations as it was a prevalence study, and the study has been led through the questionnaire, the dental examination was not conducted in the student for diagnosis of bruxism.

Moreover, a big limitation is the differences in culture and life experience between university settings in different countries. These differences suggest careful analysis in results even if they could possibly inspire further studies.

\section{Conclusion}

The study showed a strong correlation between stress and bruxism, it was also concluded that college students that showed the high level of stress suffered more with bruxism as compared to students with no stress. There was also a gender difference in the prevalence of stress. As noted, female students exhibit more stress as compared to male students.

\section{References}

1. Bulo JG, Sanchez MG (2014) Sources of Stress Among College Students. CVCITC Research Journal 1: 16-25.

2. Kania sk (2014) The relationship between gender differences and stress. Journal of Learning and Motivation 52: 92-101.

3. Pingitore G, Chrobak V, Petrie J (1991) The social and psychologic factors of bruxism. J Prosthet Dent 65: 443-446.

4. Lavigne GJ, Khoury S, Abe S, Yamaguchi T, Raphael K (2008) Bruxism physiology and pathology: An Overview for Clinicians. 35: 476-494.

5. Lobbezoo F, Ahlberg J, Glaros AG, Kato T, Koyano K, et al. (2012) Bruxism defined and graded: an international consensus. 40: 2-4.

6. Nadler SC (1957) Bruxism, a classification: Critical review. 54: 615-622.

7. Shetty S, Varun P, Babu SCL, Kumar SGP, Deepthi BC (2010) Bruxism: A Literature Review. 10: 141-148.

8. Hicks RA, Lucero-Gorman K, Bautista J, Hicks GJ (1999) Ethnicity and Bruxism. SAGE Journals 88: 240-241.

9. Brougham RR, Zail CM, Mendoza CM, Miller JR (2009) Stress, sex differences, and coping strategies among college students. Curr Psychol 28: 85-97.

10. Hanamura H, Houston F, Rylander H, Carlsson GE, Haraldson T (1987) Periodontal status and bruxism. A comparative study of patients with periodontal disease and occlusal parafunctions. 58: 173-176.

11. Pintado MR, Anderson GC, DeLong R, Douglas WH (1997) Variation in tooth wear in young adults over two-year period. 77: 313-320.

12. Kampe T, Taqdae T, Bader G, Edman G, Karlsson S (2008) Reported symptoms and clinical findings in a group of subjects with long standing bruxing behavior. J Oral Rehabil 24: 581-587.

13. Cohen S, Kamarck T, Mermelstein R (1983) A Global Measure of Perceived Stress. 24: 385-396.

14. Giraki M, Schneider C, Schäfer R (2010) Correlation between stress, stress-coping and current sleep bruxism. Head and Face Medicine 6: 1-8. 